\title{
First record of translocation in Culicidae (Diptera) mitogenomes: evidence from the tribe Sabethini
}

\author{
Camila Lorenz $^{1 * \dagger} \mathbb{D}$, João Marcelo Pereira Alves ${ }^{2 \dagger}$, Peter Gordon Foster ${ }^{3}$, Maria Anice Mureb Sallum ${ }^{1 \dagger}$ and \\ Lincoln Suesdek ${ }^{4,5+}$
}

\begin{abstract}
Background: The tribe Sabethini (Diptera: Culicidae) contains important vectors of the yellow fever virus and presents remarkable morphological and ecological diversity unequalled in other mosquito groups. However, there is limited information about mitochondrial genomes (mitogenomes) from these species. As mitochondrial genetics has been fundamental for posing evolutionary hypotheses and identifying taxonomical markers, in this study we sequenced the first sabethine mitogenomes: Sabethes undosus, Trichoprosopon pallidiventer, Runchomyia reversa, Limatus flavisetosus, and Wyeomyia confusa. In addition, we performed phylogenetic analyses of Sabethini within Culicidae and compared its mitogenomic architecture to that of other insects.

Results: Similar to other insects, the Sabethini mitogenome contains 13 protein-coding genes, 22 transfer RNA genes, two ribosomal RNA genes, and a control region. However, the gene order is not the same as that in other mosquitoes; the tyrosine $(\mathrm{Y})$ and cysteine $(\mathrm{C})$ tRNA genes have translocated. In general, mitogenome rearrangements within insects are uncommon events; the translocation reported here is unparalleled among Culicidae and can be considered an autapomorphy for the Neotropical sabethines.

Conclusions: Our study provides clear evidence of gene rearrangements in the mitogenomes of these Neotropical genera in the tribe Sabethini. Gene order can be informative at the taxonomic level of tribe. The translocations found, along with the mitogenomic sequence data and other recently published findings, reinforce the status of Sabethini as a well-supported monophyletic taxon. Furthermore, T. pallidiventer was recovered as sister to $R$. reversa, and both were placed as sisters of other Sabethini genera (Sabethes, Wyeomyia, and Limatus).
\end{abstract}

Keywords: Mitochondria, Insect, Rearrangement, Autapomorphy, Neotropical

\section{Background}

Insect mitochondrial DNA (mtDNA or the mitogenome) consists of a compact circular molecule usually $15-18 \mathrm{~kb}$ in length. It encodes 37 genes: 13 protein-coding genes (PCGs), two ribosomal RNA (rRNA) genes, and 22 transfer RNA (tRNA) genes. All of these genes are involved in translation of the PCGs [1]. This complement of 37 genes is highly conserved in metazoan animals,

\footnotetext{
* Correspondence: cammillalorenz@gmail.com

${ }^{\dagger}$ Maria Anice Mureb Sallum and Lincoln Suesdek are co senior authors and contributed equally to this study.

${ }^{+}$Camila Lorenz and João Marcelo Pereira Alves contributed equally to this study. 'Department of Epidemiology, School of Public Health, University of Sao

Paulo, Av. Dr. Arnaldo, 715, São Paulo CEP 05509-300, Brazil

Full list of author information is available at the end of the article
}

with rare exceptions [2]. The relative positions of PCGs and rRNA genes show limited variation among different phyla compared with tRNA genes [3]. For this reason, it has been proposed that tRNA gene rearrangements may be useful phylogenetic markers $[1,4]$. The ancestral insect mitogenome differs from the ancestral arthropod mitogenome only by the position of one tRNA gene [5] Although notable deviations from the ancestral insect mitogenomes in structure, gene arrangement, and gene content have been reported within insects, it is clear by analysing the numerous mitogenomes currently available that these exceptions are found only in highly derived portions of the insect phylogeny [1].

(c) The Author(s). 2019 Open Access This article is distributed under the terms of the Creative Commons Attribution 4.0 International License (http://creativecommons.org/licenses/by/4.0/), which permits unrestricted use, distribution, and reproduction in any medium, provided you give appropriate credit to the original author(s) and the source, provide a link to the Creative Commons license, and indicate if changes were made. The Creative Commons Public Domain Dedication waiver (http://creativecommons.org/publicdomain/zero/1.0/) applies to the data made available in this article, unless otherwise stated. 
Among insects, one of the most studied groups is the family Culicidae (mosquitoes) because of their medical and veterinary importance worldwide [6]. A total of 3564 species of Culicidae are currently recognised [7], classified in two subfamilies and 113 genera. There are approximately 1 hundred mitogenomes available representing several mosquito species, mainly from medically important genera of pathogen-vectoring species such as Aedes, Anopheles and Culex [8]. All currently available Culicidae mitogenomes have similar gene order and arrangement to the ancestral insect mitogenome [8-11], with the exception of an inversion reported by Beard et al. [12]. Despite their importance as transmitters of the yellow fever virus, to the best of our knowledge, there is only one study addressing the phylogenetic relationships within the tribe Sabethini, and the relationship between sabethines and other culicids using mitogenomes [13]. Although Aragão et al. [13] did not address gene arrangements within the mitogenome, there are some unique characteristics of the mitochondria of sabethines that separate them from other culicids. Members of this tribe are among the most morphologically and biologically diverse of all mosquitoes, and present greatest species diversity in the tropics [14]. In general, immature Sabethini species use as their habitat phytotelma, which can be formed by leaves, flowers, bamboo internodes, bromeliads, pitcher plants, and many other plant structures [14, 15].

Although long recognized as a monophyletic group, the taxonomic placement of sabethines within the Culicidae has varied, as well as the genus relationships within the tribe (see Judd [14]). Some advances have been made in the classification of this tribe [16-20], but the Sabethini as a whole is far from being organized into natural groups [14]. Results of the analyses corroborate the classification proposed by Judd in recognizing both the tribe Sabethini and Neotropical sabethines as monophyletic groups [21]. Currently, there are two major groups within the Sabethini, "New World" (Neotropical) and "Old World" (mainly Oriental/Australian) groups [14, 22] with approximately 700 valid species assigned into 13 genera [7]. Regarding the Sabethini taxonomic placement within Culicidae, there is considerable controversy in the literature. Harbach and Kitching [21] placed tribe Sabethini close to tribe Culicini, based on morphological characters. In contrast, Reidenbach et al. [23], using morphological and genetic data, recovered the Sabethini and Aedini tribes as closely related.

In light of these knowledge gaps and this controversial situation, we started investigating the phylogeny of $\mathrm{Cu}$ licidae at the genus level based on mitogenomes (Lorenz et al. MS submitted). Results corroborated the monophyly of Sabethini and incidentally unveiled tRNA translocations in some samples of that tribe. Then, we decided to perform a new study, now at the species level, to further understand the information obtained from sabethine mitochondrial data. The goals of the current study were to: 1) verify the arrangement and order of genes in the mitogenomes of Sabethini (Sabethes undosus, Trichoprosopon pallidiventer, Runchomyia reversa, Limatus flavisetosus, and Wyeomyia confusa) and 2) establish phylogenetic relationships among Sabethini species and with other culicids. Our results showed that the gene order contains phylogenetic information that complements the monophyly of the tribe Sabethini.

\section{Results}

General characteristics of the mitogenome of the five newly sequenced Sabethini species from this study are in Table 1. The mitochondrial genomes are similar to those of three Sabethes species studied by Aragão et al. [13], ranging from 15,302 to $16,037 \mathrm{bp}$ in length. Length variation is mainly due to differences in the length of control regions in Sabethini species. In addition to this ATrich control region, the mitogenomes also contained 37 genes: 13 PCGs, 22 tRNA genes, and two rRNA genes (Fig. 1).

Gene order in the mitogenomes in the Sabethini differed from other mosquito species. Tyrosine (Y) and cysteine (C) tRNAs are translocated (Fig. 2). In the available mitogenomes from other groups, $\mathrm{Y}$ and $\mathrm{C}$ are located between COI and tRNA-W genes, whereas in the Sabethini, they are between the tRNA-I gene and the AT control region. Our results suggest that this translocation is an autapomorphy of the Neotropical sabethines.

Results of phylogenetic analyses showed that the representative species of Sabethini are monophyletic (Fig. 3). The phylogenetic analysis based on BI and ML recovered identical tree topologies, with variations in the statistical support of some branches. The three Sabethes species available in GenBank clustered with S. undosus. The strongly supported clade composed of $T$. pallidiventer and $R$. reversa was sister to the remaining sabethines. All clades corroborate the taxonomic classification of Sabethini proposed by Reidenbach et al. [23] based on morphology and sequence data.

Regarding the translocation found in Sabethini, we searched the literature to determine whether such translocation events are common in other insect orders and mapped them onto mtDNA following Cameron [1] (Fig. 4). We represent only a simplification of few insect orders to exemplify rearrangements occurrence; Embioptera, Mantodea, and Dermaptera also present this phenomenon. There are many groups in which a given rearrangement has been observed in only a single species, but the taxonomic range of these rearrangements is currently unknown. However, it is apparent that mitogenome rearrangements within insects, with some exceptions such as hemipteroids (Phthiraptera, Psocoptera 
Table 1 General characteristics of Sabethini mitogenomes obtained in this study

\begin{tabular}{lllll}
\hline Species & Length (bp) & 1st codon AT\% ${ }^{\mathrm{a}}$ & 2nd codon AT\% & 3rd codon AT\% $^{\mathrm{a}}$ \\
\hline Sabethes undosus & 15,334 & 72.3 & 67.7 & 95.4 \\
Limatus flavisetosus & 15,663 & 72.0 & 68.4 & 96.5 \\
Wyeomyia confusa & 15,456 & 71.5 & 67.7 & 94.1 \\
Trichoprosopon pallidiventer & 16,037 & 71.0 & 67.4 & 94.8 \\
Runchomyia reversa & 15,302 & 71.7 & 67.6 & 92.8 \\
\hline
\end{tabular}

${ }^{\mathrm{a} A T \%}$ per codon position were calculated only for the 13 protein-coding genes

and Thysanoptera) and hymenopterans, are uncommon events.

\section{Discussion}

The tribe Sabethini has remarkable morphological and ecological diversity, unparalleled by other Culicidae tribes. Although widely recognized as a distinct group, the phylogenetic relationships within Sabethini are poorly known [14]. Here, we report for the first time the mitogenomes of five Sabethini species. Knowledge of the mitogenomes of these species can be useful in elucidating phylogenetic relationships within Sabethini. Mitogenomes for the newly sequenced species are similar to those of other Sabethes species studied by Aragão et al. [13]. The control region shows greater variability in length than other regions of the mitogenome, as has been observed in other Culicidae species [11]. Similar to other Culicidae [9, 24], the third codon positions has higher AT content than the first and second codons. The transcription hypothesis of codon usage proposed by Sun et al. [25] says that "the high availability of ATPs, along with the lack of other NTPs, leads to the maximization of the use of adenines in the third codon position, increasing the efficiency of the transcription". Furthermore, purifying selection against deleterious mutations is less severe on the third codon position [11]. Thus, the higher AT content in the third codon position is likely associated with biased usage within synonymous codons.

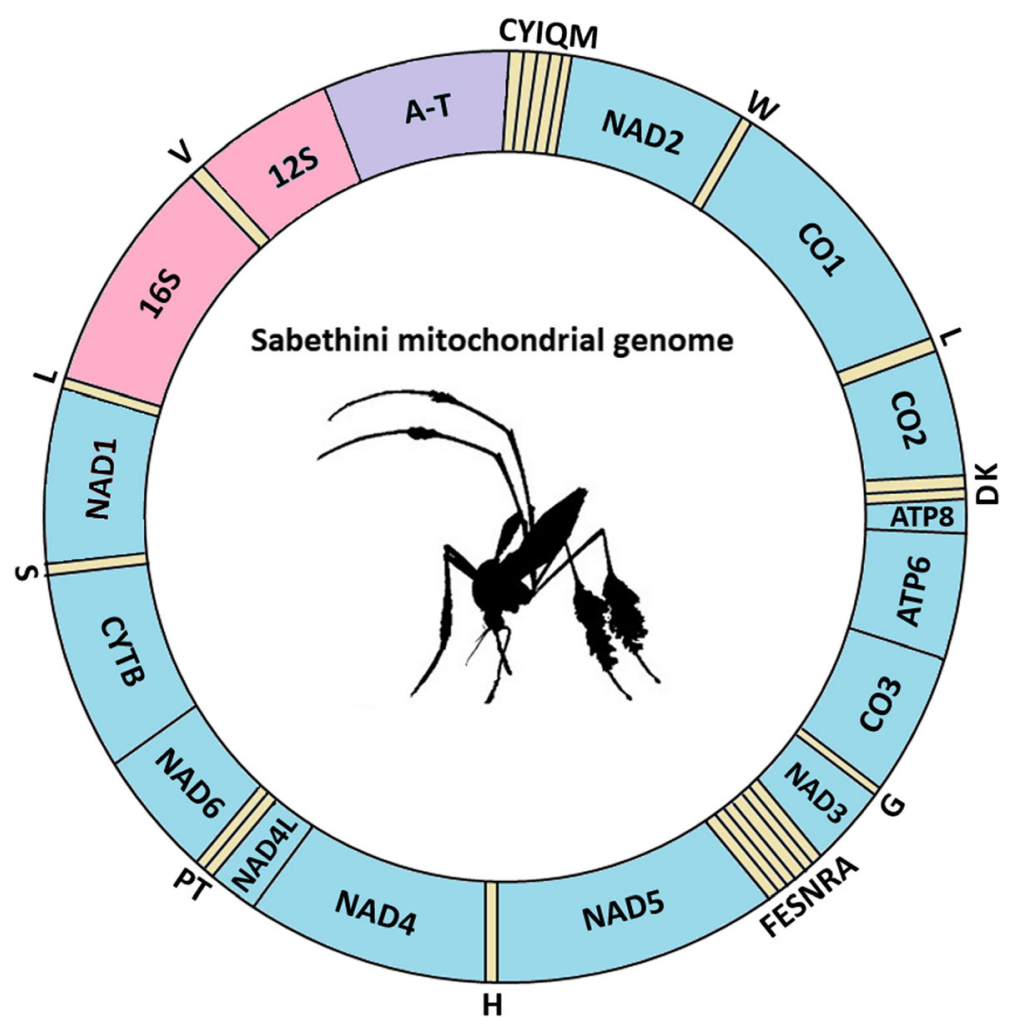

Fig. 1 Graphical representation of the arrangement and gene order of Sabethini mitochondrial genomes. The tRNA genes are indicated by letters according to the IUPAC-IUB abbreviations for amino acids. The genes in blue represent the PCGs; in pink are the ribosomal genes (small and large subunits); and in purple, the AT-rich control region 


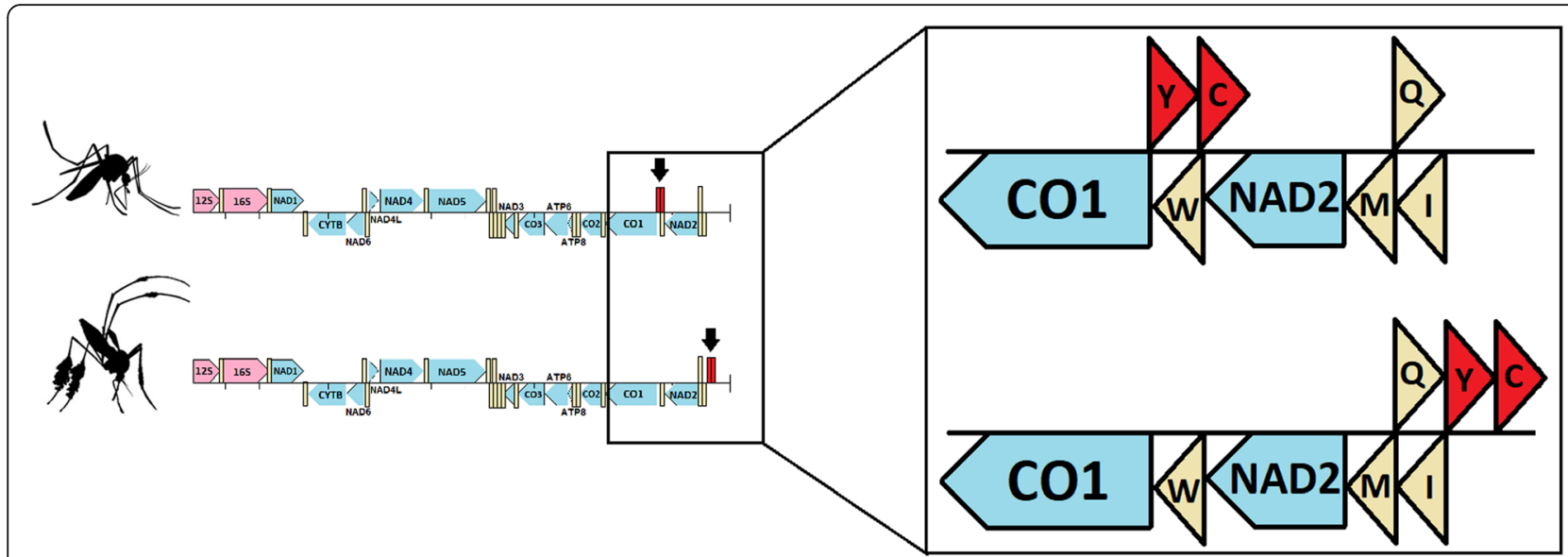

Fig. 2 Schematic diagram showing the position and orientation of mitochondrial genes: Above - most of the Culicidae mtDNA; Below representatives of the tribe Sabethini (Sabethes, Limatus, Wyeomyia, Runchomyia, and Trichoprosopon). Arrows indicate the location of C and $Y$ tRNA genes. The AT-rich control region was omitted from the representation

Regarding gene order, the translocation reported here is likely an autapomorphy of the Neotropical sabethines. This evolutionary event occurred in the Sabethini ancestor at least 75 million years ago based on the dates inferred by Reidenbach et al. [23]. Translocation events within tRNA gene regions have been found in Collembola [26], but very few gene rearrangements have been examined in invertebrates, thus it is difficult to hypothesize about the mechanisms involved in Sabethini mitogenome translocations. Tandem duplications have been proposed to produce translocations [27]. Alternatively, translocations may be produced through the illicit priming of mitochondrial replication by a tRNA molecule (originally proposed by Cantatore et al. [28]).

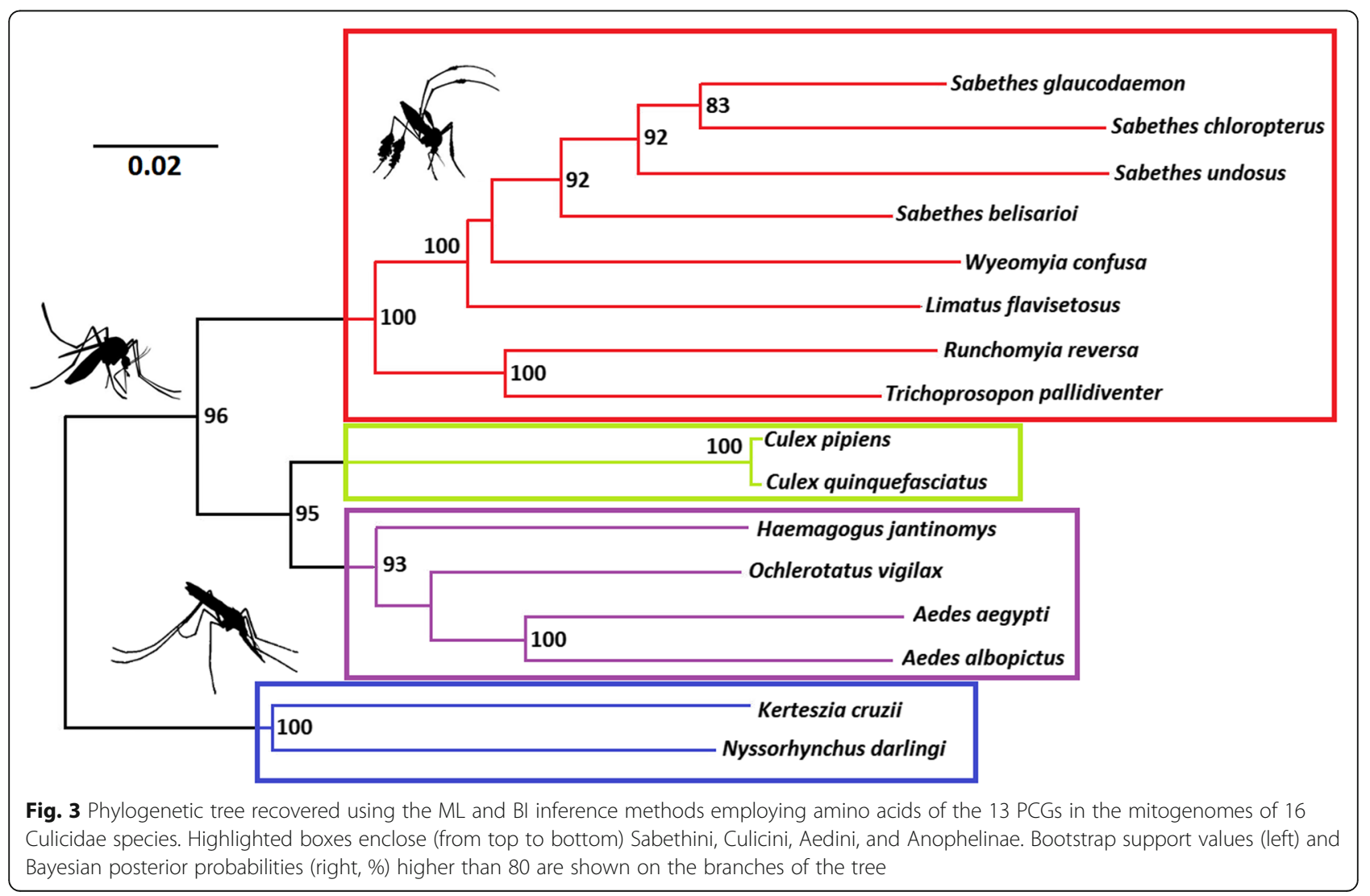




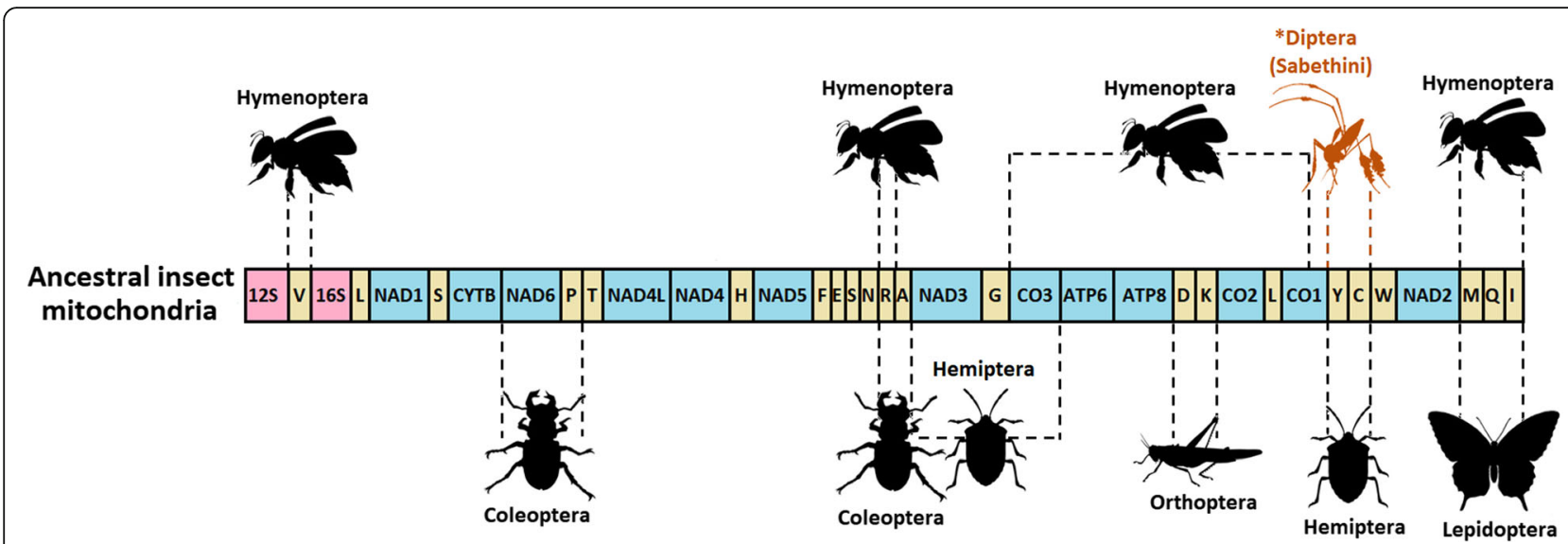

Fig. 4 Synapomorphic mitogenome rearrangements found in insect intra-orders mapped along the mitochondrial genome. The translocation of the $Y$ and $C$ tRNA genes, found in the tribe Sabethini, is highlighted in orange. The AT control region was omitted from the representation. Data regarding other insects were obtained from Cameron [1]. This graph is a simplification and do not represent all insect orders that exhibit mitochondrial rearrangements. (Images of insects available on freepik.com. Sabethini image was adapted from a photo of S. Drechsel - diptera.info)

Specifically, after mitochondrial replication is initiated, failure to cleave the tRNA primer from the nascent DNA strand could lead to the ultimate incorporation of a tRNA gene into the mitochondrial genome. It is unclear which model is more likely to have occurred in Sabethini.

Sabethini phylogenetic relationships were robustly resolved by analysis of mitogenomes (Fig. 3). The lineage composed of Trichoprosopon pallidiventer and Runchomyia reversa was sister to the group of Sabethes, Wyeomyia, and Limatus, which formed a highly supported clade. The phylogenetic tree recovered here supports those found by Reidenbach et al. [23] using six nuclear genes and 80 morphological characters. Our results are also in agreement with those of Aragão et al. [13], where the species of Sabethes were monophyletic.

Other contemporary studies of our team lead one to believe in the monophyly of Sabethini based on mitogenomes (Lorenz et al., MS submitted) and pointed to putative autapomorphic wing shape characters (Lorenz \& Suesdek, MS submitted). In addition, our study also addressed the phylogenetic placement of the genus Runchomyia using DNA sequence data for the first time. This genus is the most difficult to characterize of the sabethines studied here because no clear autapomorphies are known for this genus from any life stage [14]. Along with Runchomyia, the genus Trichoprosopon represent the most divergent branch within Sabethini, as proposed by Edwards [29]. This group has both morphological synapomorphies with Neotropical taxa and retains many of plesiomorphic characteristics of Oriental and Australian groups [14]. Regarding Sabethini phylogenetic placement within Culicidae, our findings suggest that this tribe is closer to both Culicini and Aedini than to the remaining groups studied. This finding differ from those obtained by Harbach and Kitching [21] and Reidenbach et al. [23] using distinct data sets.

All species studied here are Neotropical, and previous studies strongly support these taxa as a monophyletic group $[14,30]$. Species of the genera Trichoprosopon and Sabethes have been found to be competent to transmit arboviruses to humans and other primates [31]. The genus Wyeomyia has also been implicated in the transmission of yellow fever virus [32]. The geographical range of Sabethes, Trichoprosopon and Wyeomyia is restricted to the Neotropics. Furthermore, Sabethini from the Oriental/Australian region are not vectors of arboviruses. This suggests that vector competence in Neotropical Sabethini for arboviruses may have arisen after Gondwanan separation of the Americas and Africa [14]. The present study also indicates that vector competence to arboviruses may have arisen more than once in Culicidae, in agreement with the hypothesis proposed by Judd [14]. An alternative and parsimonious explanation is that all culicids share vectorial competence but it is expressed unequally across species.

Currently, with representative mitogenomes sequenced from each insect order, no gene rearrangements have been found to be shared between orders [1]. However, we found that synapomorphic mtDNA rearrangements can distinguish groups at taxonomic scales below the ordinal level. Rearrangements occur throughout the mitogenome, with inversions or local rearrangements being most common, whereas translocations are rare. Most gene translocations found in insect mitogenomes are useful for high-level phylogenetic reconstructions [5, 33]. Nevertheless, the results found in the present study suggest that gene order can be informative at lower 
taxonomic ranks than previously hypothesized, and reinforce the findings from sequence based phylogenetic analysis. The remaining genes in sabethine mitogenomes have the same order as the ancestral hexapodan gene arrangement [34]. Further studies addressing the mitogenomes of Sabethini will provide new insights and additional tools to investigate relationships within a group of mosquitoes of public health importance. In addition, the translocation of two tRNA genes is a remarkable phenomenon that merits investigation with broader taxon sampling.

\section{Conclusions}

In this study, we report the complete mitochondrial genomes of five Sabethini species and the occurrence of a tRNA translocation. The length of these mitogenomes was similar to that of other Sabethes species, and the AT-rich control region was the most length variable region. PCG third codon positions had a higher AT content than the first or the second codons, similar to other Culicidae species or animals in general. Regarding gene order, the translocation reported here is unprecedented among Culicidae and represents an autapomorphy for the Neotropical sabethines. Our analyses suggest that gene order can be informative at low taxonomic levels, such as tribes, reinforcing the results from sequence based phylogenetic analysis. The mitogenomes also corroborated the monophyly of Sabethini and provided robust phylogenetic support for the lineage of Trichoprosopon plus Runchomyia as sisters to a clade composed of Sabethes, Wyeomyia, and Limatus. We suggest that new insights on the phylogeny of the group could be addressed further by using larger taxon sampling, including Oriental and Australian taxa.

\section{Methods}

\section{Sample collection, data used, and DNA extraction}

Mosquito species used in this study share similar habitats, and were captured in the same region of Atlantic forest, São Paulo state, Brazil (245' ${ }^{\prime}$ S, $\left.47^{\circ} 51^{\prime} \mathrm{W}\right)$, except for T. pallidiventer, which was captured in the Amazon forest, Amazonas, Brazil $\left(3^{\circ} 05^{\prime} \mathrm{S}, 60^{\circ} 00^{\prime} \mathrm{W}\right)$. Fieldcollected specimens were identified to species level using morphological keys of Forattini [35] and Lane [36]. Adult females were collected and stored at $-80^{\circ} \mathrm{C}$ until DNA extraction. Genomic DNA was extracted from each mosquito individually using a Qiagen DNeasy ${ }^{\circ}$ Blood and Tissue Kit (Qiagen Ltd., Crawley, UK) following the same procedure as Foster et al. [37]. DNA was examined after electrophoresis on 1\% agarose gels and used for PCR amplification. A list of all species used in this study is in Table 2 .
Table 2 Species used in this study and their mitogenome GenBank accession numbers

\begin{tabular}{lll}
\hline Species & References & GenBank \\
\hline Sabethes undosus & This study & MK575488 \\
Trichoprosopon pallidiventer & This study & MK575490 \\
Runchomyia reversa & This study & MK575487 \\
Limatus flavisetosus & This study & MK575482 \\
Wyeomyia confusa & This study & MK575492 \\
Sabethes chloropterus & {$[13]$} & MF957172 \\
Sabethes glaucodaemon & {$[13]$} & MF957173 \\
Sabethes belisarioi & {$[13]$} & MF957171 \\
Aedes aegypti & {$[38]$} & MF194022 \\
Aedes albopictus & {$[39]$} & KR068634 \\
Culex quinquefasciatus & {$[12]$} & GU188856 \\
Culex pipiens & {$[40]$} & KT851543 \\
Ochlerotatus vigilax & {$[41]$} & KP721463 \\
Haemagogus janthinomys & {$[42]$} & KT372555 \\
Nyssorhynchus darlingi & {$[43]$} & GQ918272 \\
Kerteszia cruzii & {$[10]$} & KU551289 \\
\hline According Foster et al. & &
\end{tabular}

${ }^{\mathrm{a}}$ According to Foster et al. [44]

\section{PCR amplification and sequencing}

The mitochondrial genome of each individual was amplified using the one-step long-range polymerase chain reaction method [45]. Reactions were prepared as follows: $25 \mu \mathrm{L}$ of GoTaq Long PCR Master Mix 2x (Promega, $\mathrm{WI}), 0.3 \mu \mathrm{M}$ of each primer (forward and reverse), $1-$ $2 \mu \mathrm{L}$ of extracted DNA, and sterile water to make up final volume of $50 \mu \mathrm{L}$. The primers used for mitochondrial genome amplification follow Hwang et al. [45]: HPK16Saa for the forward strand and HPK16Sbb for the reverse strand. The size of the amplified fragment was approximately $15,300 \mathrm{bp}$. Thermocycler conditions consisted of: initial denaturation at $94{ }^{\circ} \mathrm{C}$ for $2 \mathrm{~min}$, followed by 39 cycles of $94{ }^{\circ} \mathrm{C}$ for $15 \mathrm{~s}$, annealing at $65^{\circ} \mathrm{C}$ for $20 \mathrm{~s}$, and $65^{\circ} \mathrm{C}$ for $15 \mathrm{~min}$, and one final extension cycle at $72^{\circ} \mathrm{C}$ for $10 \mathrm{~min}$. The only step that varied between species was annealing temperature: $65^{\circ} \mathrm{C}$ for Wyeomyia confusa and Trichoprosopon pallidiventer, $62^{\circ} \mathrm{C}$ for Limatus flavisetosus, and $55^{\circ} \mathrm{C}$ for Sabethes undosus and Runchomyia reversa. Amplicons were purified using a DNA Clean \& Concentrator kit (Zymo Research, CA) and quantified using a Qubit 2.0 fluorometer (Life Technologies, OR).

\section{Mitogenome sequence assembly and analysis}

Next-generation sequencing was employed to obtain mitochondrial DNA sequences of five Sabethini species. Barcoded libraries were constructed from the PCR products using the Nextera XT DNA Sample Preparation Kit (Illumina, IL) and sequenced on the Illumina MiSeq 
platform with paired-end $250 \mathrm{bp}$ read chemistry. Raw nucleotide sequences were checked using FastQC software [46]. Mitochondrial genomes were assembled using Mira v4 [47] and Newbler v2.9 software, and visualized in Tablet [48]. Mitochondrial genes were annotated using MITOS [49]. DNA and amino acid (AA) sequence alignments were performed with MEGA 6.06 software [50] and the ClustalW algorithm. Phylogenetic inferences were made using the 13 mitochondrial PCGs from the five Sabethini species sequenced here and other $\mathrm{Cu}$ licidae available on GenBank (see Table 2). To perform a more comprehensive phylogenetic analysis of the tribe Sabethini, in addition to the newly sequenced samples from our study, we also included in our analysis three species of Sabethes previously sequenced by Aragão et al. [13]: Sabethes chloropterus (MF957172), Sabethes glaucodaemon (MF957173), and Sabethes belisarioi (MF957171). We tested all of the following dataset combinations: amino acids, all DNA sites, or 1st and 2nd codon positions alone. All tests produced the same topology, differing only nodal support. Sequences were concatenated using FASconCAT-G v. 1.04 [51]. All phylogenetic analyses were partitioned based on gene, with gamma-distributed site heterogeneity, and substitution models estimated independently for each partition. Phylogenetic analysis was performed using Maximum Likelihood (ML) with bootstraping $=1000$ in RAxML v8.2.11 software [52]. Bayesian phylogenetic inference (BI) was conducted using MrBayes v. 3.2.6 [53] with four runs of eight chains each, run for at least 5 million generations. The tree generated was visualized and customized in FigTree v1.4.3 software [54].

\section{Acknowledgements}

Not applicable.

\section{Authors' contributions \\ $\mathrm{CL}, \mathrm{MAMS}$ and $\mathrm{LS}$ designed the study. $\mathrm{CL}$ were responsible for mitochondrial genome amplification and literature search. JMPA analyzed the Illumina data and did the assembly of the mitochondrial genes. CL and MAMS wrote the draft with the help of PGF, JMPA and LS. All authors read and approved the final version of the manuscript.}

\section{Funding}

LS has been fellow of Conselho Nacional de Desenvolvimento Científico e Tecnológico (CAPES) grants 311805/2014-0 and 311984/2018-5. CL was granted by fellowships no. 2013/05521-9 and 2015/12784-1 from São Paulo Research Foundation (FAPESP). MAMS is financially supported by São Paulo Research Foundation (FAPESP) grant no. 2014/26229-9 and CNPq no. $301877 / 2016-5$. The funders had no role in study design, data collection and analysis, decision to publish, or preparation of the manuscript.

\section{Availability of data and materials}

All the nucleotide data generated in this study were submitted to the NCB sequence nucleotide database under the accession numbers: MK575482, MK575487, MK575488, MK575490, and MK575492.

\section{Ethics approval and consent to participate} Not applicable.
Consent for publication

Not applicable.

\section{Competing interests}

The authors declare that they have no competing interests.

\section{Author details}

'Department of Epidemiology, School of Public Health, University of Sao Paulo, Av. Dr. Arnaldo, 715, São Paulo CEP 05509-300, Brazil. ²Department of Parasitology, Institute of Biological Science, University of Sao Paulo, Av. Prof. Lineu Prestes, 1374, São Paulo, SP 05508-000, Brazil. ${ }^{3}$ Department of Life Sciences, Natural History Museum, Cromwell Rd, London, UK. ${ }^{4}$ Butantan Institute, Av. Vital Brazil 1500, Butanta, São Paulo, SP CEP 05503-900, Brazil. ${ }^{5}$ Institute of Tropical Medicine, University of Sao Paulo, Av. Dr. Enéas de Carvalho Aguiar 470, Jardim América, São Paulo, SP CEP 05403-000, Brazil.

Received: 12 March 2019 Accepted: 29 August 2019

Published online: 27 September 2019

\section{References}

1. Cameron SL. Insect mitochondrial genomics: implications for evolution and phylogeny. Annu Rev Entomol. 2014;59:95-117.

2. Boore JL. Animal mitochondrial genomes. Nucleic Acids Res. 1999;27:176780.

3. Päbabo S, Thomas WK, Whitfield KM, Kumazawa Y, Wilson AC. Rearrangements of mitochondrial transfer RNA genes in marsupials. J Mol Evol. 1991;33:426-30

4. Macey JR, Larson A, Ananjeva NB, Fang Z, Papenfuss TJ. Two novel gene orders and the role of light-strand replication in rearrangement of the vertebrate mitochondrial genome. Mol Biol Evol. 1997;14:91-104.

5. Boore JL, Lavrov DV, Brown WM. Gene translocation links insects and crustaceans. Nature. 1998;392:660-7.

6. Foster WA, Walker ED. Mosquitoes (Culicidae). In Medical and veterinary Entomology: Academic Press; 2019

7. Mosquito Taxonomic Inventory. 2019. http://mosquito-taxonomic-inventory. info/. Accessed 20 May 2019

8. GenBank in National Center for Biotechnology Information. 2018. https:// www.ncbi.nIm.nih.gov/. Accessed 13 Dec 2018.

9. Demari-Silva B, Foster PG, de Oliveira TM, Bergo ES, Sanabani SS, Pessôa R, Sallum MAM. Mitochondrial genomes and comparative analyses of Culex camposi, Culex coronator, Culex usquatus and Culex usquatissimus (Diptera: Culicidae), members of the coronator group. BMC Genomics. 2015;16:831.

10. Oliveira TMP, Foster PG, Bergo ES, Nagaki SS, Sanabani SS, Marinotti O, Sallum MAM. Mitochondrial genomes of Anopheles (Kerteszia)(Diptera: Culicidae) from the Atlantic forest, Brazil. J Med Entomol. 2016;53:790-7.

11. Hao YJ, Zou YL, Ding YR, Xu WY, Yan ZT, Li XD, Chen B. Complete mitochondrial genomes of Anopheles stephensi and An. dirus and comparative evolutionary mitochondriomics of 50 mosquitoes. Sci Rep. 2017;7(1):7666

12. Beard CB, Hamm DM, Collins FH. The mitochondrial genome of the mosquito Anopheles gambiae: DNA sequence, genome organization, and comparisons with mitochondrial sequences of other insects. Insect Mol Biol. 1993:2:103-24

13. Aragão A, Neto JPN, Cruz ACR, Casseb SMM, Cardoso JF, da Silva SP, Ishikawa EAY. Description and phylogeny of the mitochondrial genome of Sabethes chloropterus, Sabethes glaucodaemon and Sabethes belisarioi (Diptera: Culicidae). Genomics. 2018;6:1-8.

14. Judd D. Review of the systematics and phylogenetic relationships of the Sabethini (Diptera: Culicidae). Syst Entomol. 1996;21:129-50

15. Frank JH, Lounibos LP. Phytotelmata: swamps or islands? Fla Entomol. 1987; 70:14-20.

16. Zavortink TJ. Mosquito studies (Diptera, Culicidae). XXXV. The new sabethine genus Johnbelkinia and a preliminary reclassification of the composite genus Trichoprosopon. Contr Am Ent Inst. 1979;12:1-61.

17. Mattingly PF. Medical entomology studies the subgenera Rachiontomyia Tricholeptomyia and Tripteroide in the oriental region (Diptera: Culicidae). Contrib Am Ent Inst. 1981;17:1-147.

18. Harbach RE, Peyton EL. A new subgenus of Wyeomyia (Diptera: Culicidae) with the reclassification and redescription of Wyeomyia (Davismyia) arborea, Wyeomyia (Dendromyia) tarsata and Sabethes (Sabethes) carrilloi. Mosq Syst. 1991;23:92-109. 
19. Harbach RE, Peyton EL. Morphology and evolution of the larval maxilla and its importance in the classification of the Sabethini (Diptera: Culicidae). Mosq Syst. 1993;25:1-16.

20. Motta MA, Lourenço-de-Oliveira R. Wyeomyia luteoventralis Theobald, the type species of the subgenus Dendromyia Theobald (Diptera: Culicidae). Mem Inst Osw Cruz. 1995;90:375-85.

21. Harbach RE, Kitching IJ. Phylogeny and classification of the Culicidae (Diptera). System Entomol. 1998;23:327-70.

22. Ward RA. Third supplement to 'A catalog of the mosquitoes of the World' (Diptera: Culicidae). Mosq Syst. 1992;24:177-230.

23. Reidenbach KR, Cook S, Bertone MA, Harbach RE, Wiegmann BM, Besansky NJ. Phylogenetic analysis and temporal diversification of mosquitoes (Diptera: Culicidae) based on nuclear genes and morphology. BMC Evol Biol. 2009;9:298-302

24. Krzywinski J, Li C, Morris M, Conn JE, Lima JB, Povoa MM, Wilkerson RC. Analysis of the evolutionary forces shaping mitochondrial genomes of a Neotropical malaria vector complex. Mol Phylogenet Evol. 2011;58:469-77.

25. Sun WY, Xu DL, Chen HX, Shi W, Sundberg P, Strand M, Sun SC. Complete mitochondrial genome sequences of two parasitic/commensal nemerteans, Gononemertes parasita and Nemertopsis tetraclitophila (Nemertea: Hoplonemertea). Parasit Vectors. 2014:7:273.

26. Nardi F, Carapelli A, Fanciulli PP, Dallai R, Frati F. The complete mitochondrial DNA sequence of the basal hexapod Tetrodontophora bielanensis: evidence for heteroplasmy and tRNA translocations. Mol Bio Evol. 2001;18:1293-304.

27. Dowton M, Austin AD. Evolutionary dynamics of a mitochondrial rearrangement" hot spot" in the hymenoptera. Mol Biol Evol. 1999;16:298-309.

28. Cantatore P, Gadaleta MN, Roberti M, Saccone C, Wilson AC. Duplication and remoulding of tRNA genes during the evolutionary rearrangement of mitochondrial genomes. Nature. 1987;329:853.

29. Edwards FW. Diptera: Fam. Culicidae. Gen. Ins. 1932;194:30-67.

30. Harbach RE. The Culicidae (Diptera): a review of taxonomy, classification and phylogeny. Zootaxa. 1668;2007:591-638.

31. Mattingly PF. Tr.(Tripteroides). Mosq Syst. 1974;6:231

32. Galindo P, Trapido H. Forest mosquitoes associated with sylvan yellow fever in Nicaragua. Am J Trop Med Hyg. 1957;6:145-52.

33. Boore JL, Brown WM. Big trees from little genomes: mitochondrial gene order as a phylogenetic tool. Curr Opin Genet Dev. 1998;8:668-74.

34. Crease TJ. The complete sequence of the mitochondrial genome of Daphnia pulex (Cladocera: Crustacea). Gene. 1999:233:89-99.

35. Forattini OP. Culicidologia médica: identificaçäo, biologia e epidemiologia. In: Culicidologia Médica: Identificaçäo, Biologia E Epidemiologia, vol. 2. In press; 2002.

36. Lane J. Neotropical Culicidae, vol. 1. Sao Paulo, In press: University of Sao Paulo; 1953

37. Foster PG, Bergo ES, Bourke BP, Oliveira TM, Nagaki SS, Sant'Ana DC, Sallum MAM. Phylogenetic analysis and DNA-based species confirmation in Anopheles (Nyssorhynchus). PLoS One. 2013:8:e54063.

38. Matthews BJ, Dudchenko O, Kingan SB, Koren S, Antoshechkin I, Crawford JE, Weedall GD. Improved reference genome of Aedes aegypti informs arbovirus vector control. Nature. 2018:563:501-9.

39. Zhang H, Xing D, Wang G, Li C, Zhao T. Sequencing and analysis of the complete mitochondrial genome of Aedes albopictus (Diptera: Culicidae) in China. Mitochondr Dna Part A. 2016;27:2787-8.

40. Luo QC, Hao YJ, Meng F, Li TJ, Ding YR, Hua YQ, Chen B. The mitochondrial genomes of Culex tritaeniorhynchus and Culex pipiens pallens (Diptera: Culicidae) and comparison analysis with two other Culex species. Parasit Vectors. 2016;9:406.

41. Hardy CM, Court LN, Morgan MJ. The complete mitochondrial DNA genome of Aedes vigilax (Diptera: Culicidae). Mitochondr Dna Part A. 2016; 27:2552-3.

42. Lemos PDS, Monteiro HADO, Castro FC, Lima CPSD, Silva DEAD, Vasconcelos JMD, Nunes MRT. Characterization of mitochondrial genome of Haemagogus janthinomys (Diptera: Culicidae). Mitochondr Dna Part A. 2017; 28:50-1.

43. Moreno M, Marinotti O, Krzywinski J, Tadei WP, James AA, Achee NL, Conn JE. Complete mtDNA genomes of Anopheles darlingi and an approach to anopheline divergence time. Malaria J. 2010;9:127.

44. Foster PG, de Oliveira TMP, Bergo ES, Conn JE, Sant'Ana DC, Nagaki SS, Sallum MAM. Phylogeny of Anophelinae using mitochondrial protein coding genes. Roy Soc Open Sci. 2017:4:170758.
45. Hwang UW, Park CJ, Yong TS, Kim W. One-step PCR amplification of complete arthropod mitochondrial genomes. Mol Phylogenet Evol. 2001;19: 345-52.

46. Andrews S. FastQC: quality control tool for high throughput sequence data. 2010. http://www.bioinformatics.babraham.ac.uk/projects/fastqc. Accessed 20 Oct 2018.

47. Chevreux B, Wetter T, Suhai S. Genome sequence assembly using trace signals and additional sequence information. Comput Sci Biol Proc. 1999:99:45-56.

48. Milne I, Bayer M, Cardle L, Shaw P, Stephen G, Wright F, Marshall D. Tablet - next generation sequence assembly visualization. Bioinformatics. 2009;26:401-2.

49. Bernt M, Donath A, Jühling F, Externbrink F, Florentz C, Fritzsch G, Stadler PF. MITOS: improved de novo metazoan mitochondrial genome annotation. Mol Phylogenet Evol. 2013;69:313-9.

50. Tamura K, Stecher G, Peterson D, Filipski A, Kumar S. MEGA6: molecular evolutionary genetics analysis version 6.0. Mol Biol Evol. 2013;30:2725-9.

51. Kück P, Meusemann K. FASconCAT: convenient handling of data matrices. Mol Phylogenet Evol. 2010;56:1115-8.

52. Stamatakis A. RAxML version 8: a tool for phylogenetic analysis and postanalysis of large phylogenies. Bioinformatics. 2014;30:1312-3.

53. Ronquist F, Teslenko M, Van Der Mark P, Ayres DL, Darling A, Höhna S, Huelsenbeck JP. MrBayes 3.2: efficient Bayesian phylogenetic inference and model choice across a large model space. Syst Biol. 2012;61:539-42.

54. Rambaut A. FigTree version 1.4.0. 2016. http://tree.bio.ed.ac.uk/software/ figtree. Accessed 10 Oct 2018.

\section{Publisher's Note}

Springer Nature remains neutral with regard to jurisdictional claims in published maps and institutional affiliations.

Ready to submit your research? Choose BMC and benefit from:

- fast, convenient online submission

- thorough peer review by experienced researchers in your field

- rapid publication on acceptance

- support for research data, including large and complex data types

- gold Open Access which fosters wider collaboration and increased citations

- maximum visibility for your research: over $100 \mathrm{M}$ website views per year

At BMC, research is always in progress.

Learn more biomedcentral.com/submissions 